\title{
Chief Medical Officer bei Sportverbänden
}

\section{Tanja Kühnle}

Dipl.-Biol., Managing Editor

Die letzte Prüfung ist geschafft, das Medizinstudium beendet. Und wie geht es jetzt weiter? In unserer Serie «Du findest Deinen Weg!» stellen wir Ihnen in unregelmässigen Abständen aussergewöhnliche Berufsperspektiven für Mediziner vor. In dieser Ausgabe berichtet Dr. Patrik Noack von seiner Tätigkeit als Verbandsarzt.

\section{Herr Dr. Noack, wie kam es, dass Sie Verbandsarzt geworden sind?}

Ich war schon immer sportbegeistert und habe während meines Medizinstudiums schnell gemerkt, dass der Operationssaal nichts für mich ist. Mich interessierte die konservative Orthopädie. Also machte ich meinen Facharzt in Innerer Medizin und zusätzlich den Fähigkeitsausweis Sportmedizin. Über meine Frau kam ich dann zum Triathlonsport. Zufällig war ein damaliger Kollege dort Verbandsarzt. Ich bin dann 2005 hinzugestossen. Und es folgte auch gleich der erste Einsatz in Gamagōri, Japan, wo Nicola Spirig bei den U23Weltmeisterschaften den dritten Rang belegte. Das war ein toller Start! Mittlerweile betreue ich auch noch die Skilangläufer, Swiss Sliding, Swiss Cycling und - was mich besonders freut, da ich selbst lange diese Sportart betrieben habe - die Leichtathleten.

\section{Sind Sie ausschliesslich als Verbandsarzt tätig?}

Nein, hauptsächlich arbeite ich als Allgemein- und Sportmediziner im «Zentrum für Medizin \& Sport» in Abtwil. Das Zentrum gehört zur Medbase-Gruppe, und wir arbeiten dort in einem interdisziplinären Team bestehend aus Allgemein- und Sportmedizinern, diversenFachärzten, Physiotherapeuten und Therapeuten aus dem Bereich der Komplementärmedizin

Welche Aufgaben nehmen Sie als Verbandsarzt wahr? Die Hauptarbeit ist nicht während eines Wettkampfes, sondern unter dem Jahr zu erledigen. Beispielsweise organisiere und überwache ich die jährliche sportärztliche Untersuchung der Athleten. Ausserdem bespreche ich am Ende einer Saison mit jedem, was gut und was schlecht lief und was man besser machen kann. Bei Bedarf stelle ich dann auch ein Übungsprogramm auf, da-

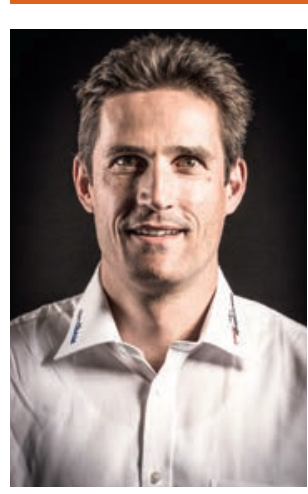

Name: Dr. Patrik Noack

Alter: 43

Zivilstand: verheiratet,

zwei Kinder

Als Verbandsarzt tätig seit: Swiss Triathlon 2005, Swiss Ski Langlauf 2007, Swiss Sliding 2012, Swiss Cycling 2014, Swiss Athletics 2015

Funktion: Chief Medical Officer Olympische Winterspiele 2018 Ausbildung: Facharzt für Allgemeine Innere Medizin, Fähigkeitsausweis Sportmedizin, Manuelle Medizin und Ultraschall Abdomen

mit der Sportler nach der Pause wieder beschwerdefrei starten kann. Im Blut schaue ich zudem nach einem Eisen-, Vitamin- B $_{12}$ oder Vitamin-D-Mangel. Und ich berate die Sportler, was die Einnahme von Supplementen und Neuerungen auf der Dopingliste betrifft. Schliesslich plane ich als Leiter des Medical-Teams, welcher Arzt und welcher Physiotherapeut zu welchen Wettkämpfen reist. Ich bin nicht jedes Mal persönlich dabei.

\section{Und welche Aufgaben übernehmen Sie während eines Wettkampfes?}

Dann stehe ich mit einem gut ausgestatteten Rucksack im Start- und Zielbereich. Denn ich muss nicht nur kleine Wunden erstversorgen, sondern beispielsweise auch Fremdkörper aus dem Auge entfernen oder Nasenbluten behandeln können. Bei schwerwiegenden Verletzungen wie zum Beispiel einem Knochenbruch suche ich mit dem Sportler einen Medical-Posten auf und schaue dort, wie es dann weitergeht. Bei einem Wettkampf ist das Unvorhergesehene die Herausfor- 


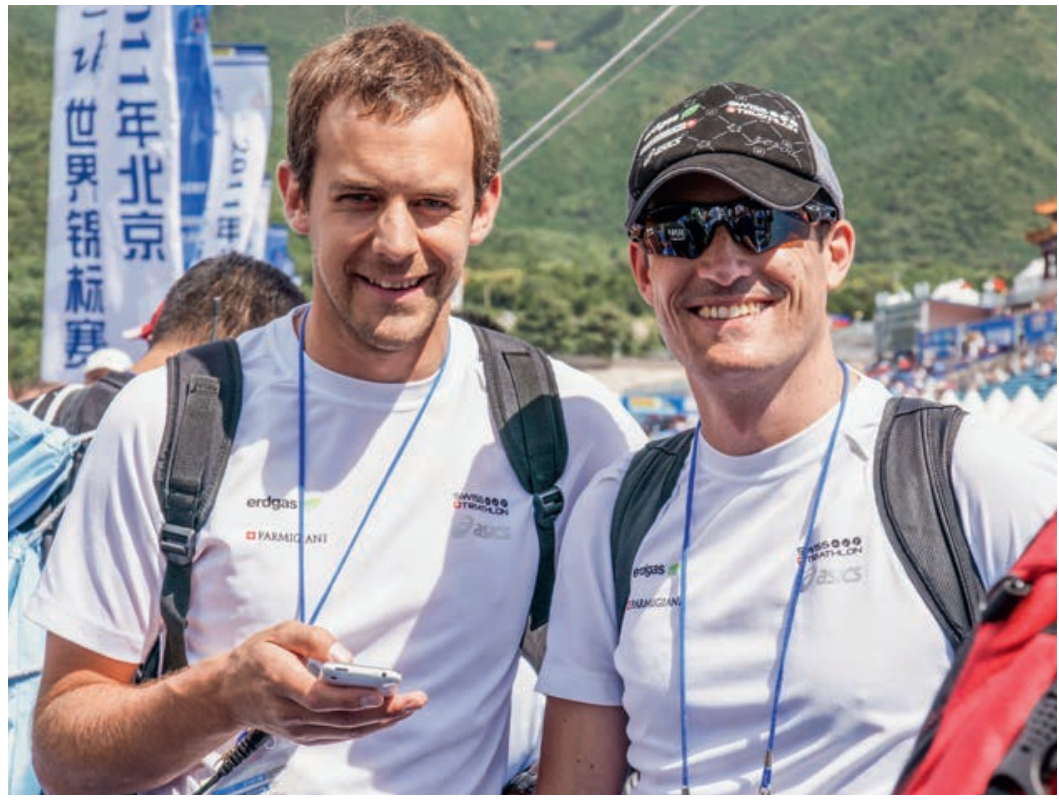

Patrik Noack (rechts) und Dominik Pürro, damals Office Manager Swiss Triathlon, an der Triathlonstrecke in Peking.

derung, nicht unbedingt das Fachliche. Hier zählt schnelle Erreichbarkeit und das schnelle Aufgleisen von Abklärungen. Das ist nicht immer ganz einfach insbesondere im Ausland.

\section{Können Sie ein Beispiel nennen?}

In Sotschi war ich mit einem Sportler wegen des Verdachts auf eine Enteritis in einem russischen Spital. Leider sprach der Chirurg dort kaum Englisch. Also hat ein Volontär, der nicht medizinisch gebildet war, übersetzt. Der Chirurg sagte: «We need to look in», weil er eine Appendizitis vermutete. Ich wollte jedoch erst einmal ein CT haben. Also mussten wir das organisieren. Und weil das Fachpersonal vor Ort nicht so gut ausgebildet war, habe ich die Bilder via Internet zu einem Kollegen in die Schweiz geschickt und von ihm beurteilen lassen.

\section{Dürfen Sie während eines Wettkampfes gleich zum Verletzten in die Arena laufen?}

Bei Olympischen Spielen ist immer das Feldteam des Organisators für die medizinische Versorgung verantwortlich und man wird dann als Mannschaftsarzt sozusagen eingeladen. Bei Weltmeisterschaften ist es nicht so klar geregelt. Da muss man vor Ort schauen, wie die Versorgung organisiert ist. Am besten stellt man sich dem Team dort schon einmal vor, dann wird man meist schnell dazugerufen, wenn etwas passiert ist. Auch für die Sportler ist es sehr beruhigend, wenn sie im Falle eines Unfalls gleich ein bekanntes Gesicht sehen.

Betreuen Sie die Sportler auch in der Zwischensaison? Ja. Für manche übernehme ich sogar ein bisschen die
Hausarztfunktion. Und wenn sich jemand im Training verletzt, ruft man mich meistens an und ich organisiere dann die Abklärung - gegebenenfalls auch bei einem meiner Kollegen in Abtwil oder bei einem Arzt in einem anderen Kanton. Das ist Dank einer schweizweiten Vernetzung bei Medbase sowie eines sportmedizinischen Netzwerkes an sich gut möglich.

\section{Klingelt das Telefon manchmal auch nachts?}

Eher selten. Aber wenn ein Athlet zum Beispiel in Dubai stürzt, dann kommt das aufgrund der Zeitverschiebung schon mal vor.

\section{Wie kommunizieren Sie dann mit den Verantwort- lichen vor Ort?}

Über eine spezielle, passwortgeschützte Online-Plattform, auf der wir die Daten ablegen und auf die sowohl die Physiotherapeuten und Ärzte als auch die Athleten Zugriff haben. Und natürlich stehen wir auch immer mit den zuständigen Hausärzten und Sportmedizinern in der Schweiz in Kontakt.

\section{Was ist, wenn ein Athlet auf einem Wettkampf einen Infekt bekommt?}

Dann isolieren wir den Athleten. Schliesslich wäre es fatal, wenn er das ganze Team ansteckt. Da die Sportler meist zu zweit auf einem Zimmer wohnen, muss der Teamchef dann schnell für ein paar Tage ein Einzelzimmer organisieren. Je nach Schweregrad der Erkrankung müssen wir dem Athleten auch manchmal zur Abreise raten. Das sind dann harte und schwierige Diskussionen - schliesslich hat der Sportler viele Monate auf diesen Einsatz hingearbeitet.

\section{Unternehmen Sie auch schon etwas im Vorfeld, um solchen Infekten vorzubeugen?}

Ja, im Winter ist die Prävention ein grosses Thema. Wir haben meist Luftbefeuchter dabei, um ein Austrocknen der Atemwege zu verhindern. Zudem empfehlen wir wir Salzwasser-Nasenspülungen und -inhalationen, Nasensalbe sowie Phytotherapeutika (Echinacea und Holunder) und Mineralstoffe (Zink).

\section{Gibt es auch Erkrankungen, mit denen die Athleten verstärkt im Sommer zu kämpfen haben?}

Im Sommer haben viele Probleme mit Allergien und Asthma. Wir beraten die Sportler dann, welche Medikamente sie nehmen dürfen, und manchmal empfehlen wir auch eine Desensibilisierung.

\section{Gab es bei einem Wettkampf schon einmal eine besonders kritische Situation?}

An der Triathlon-EM 2006 in Lausanne kam ein Schwei- 


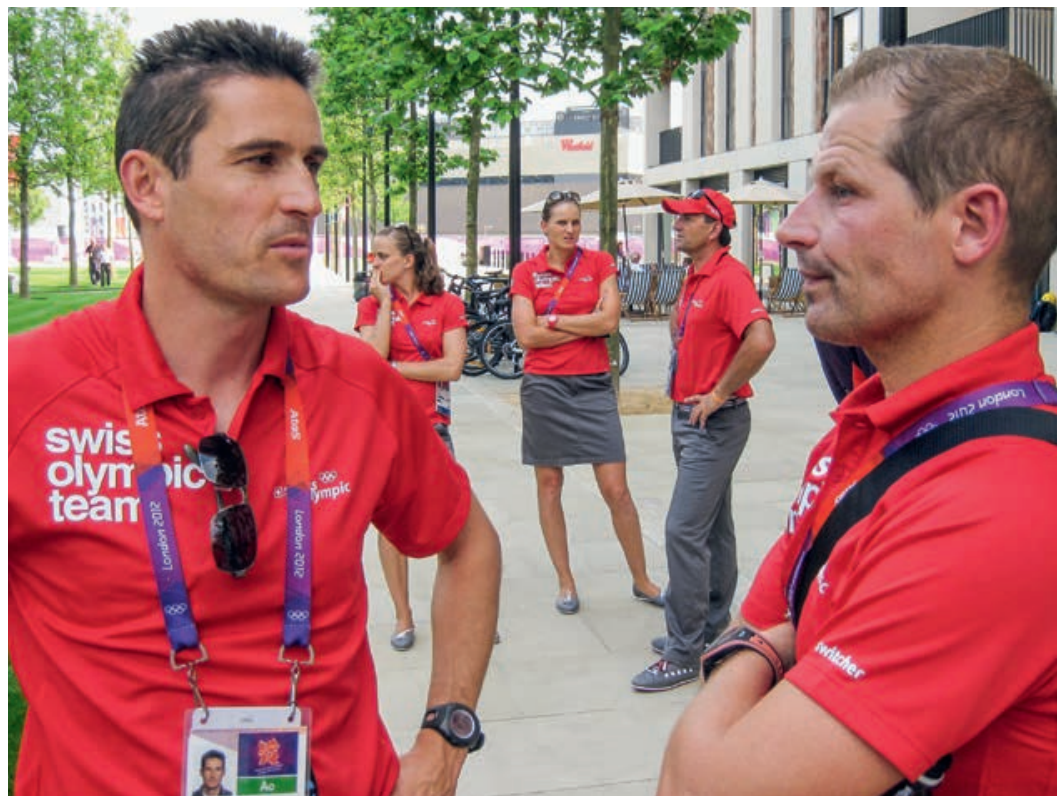

Olympische Spiele 2012 in London: Patrik Noack (links) im Gespräch mit Sportpsychologe Jörg Wetze.

zer Athlet schwankend ins Ziel aufgrund Dehydratation. Am schlimmsten ist es aber, wenn ein Triathlet oder Velofahrer nach einem Sturz am Boden liegen bleibt.

\section{Was war Ihre bislang grösste Herausforderung?}

Das war eine komplizierte Verletzung von Dario Cologna. Er hatte sich am 11. November 2013, drei Monate vor den Olympischen Spielen, einen Innen- und Aussenbandriss sowie einen Syndesmosebandriss am rechten Fuss zugezogen. Da war uns allen klar, dass es sehr knapp mit einer Teilnahme wird. Dank eines super Teamworks konnte er tatsächlich starten und zwei Goldmedaillen gewinnen. Das war die grösste Herausforderung und retrospektiv auch die schönste.

\section{Gibt es einen Sportler, den Sie gerne mal betreuen würden?}

Ja, Roger Federer ist für mich ein grosses Idol. Ich habe ihm bei den Olympischen Spielen in London bei seinem Besuch des Schweizer Teams im Olympischen Dorf kurz kennengelernt. Er kam inkognito mit tief ins Gesicht gezogener Kappe ins Dorf, aber jemand hatte ihn erkannt. Und sofort hat sich eine grosse Menschentraube um ihn herum gebildet. Das ist auch der Grund, wieso er nie im Olympischen Dorf wohnt.

\section{Wenn Sie als Verbandsarzt zu einer Veranstaltung reisen, kommt Ihre Familie dann mit?}

Als wir noch keine Kinder hatten, war meine Frau auch mal im Triathlon-Trainingslager dabei und hat an den Velotrainings teilgenommen. Momentan reise ich meist allein. Für meine Kinder, sie sind fünf und sieben Jahre alt, wäre es eher langweilig, da ich mich ja auf meine Arbeit konzentrieren muss. Ausserdem müssten wir beispielsweise bei den Olympischen Spielen getrennt wohnen, da nur ich eine Akkreditierung habe und im Olympischen Dorf wohnen darf.

\section{Was fasziniert Sie an der Verbandsarzt-Tätigkeit am meisten?}

Es ist für mich faszinierend, zu sehen, was ein menschlicher Körper leisten kann und wie die Sportler mit dem immensen Druck umgehen. Ich sage immer: Ich mache diesen Job so lange, wie ich in Wettkampfsituationen noch Hühnerhaut bekomme. Und das ist immer noch der Fall: Ich fiebere mit jedem Athleten mit!

\section{Ist Ihr Beruf gut mit der Familie vereinbar?}

Ja. Klar gibt es Situationen, wo es schön wäre, wenn man zu Hause sein könnte. Aber so häufig bin ich nun auch wieder nicht unterwegs. Ausserdem war meiner Frau und mir früh klar, dass die Verbandsarzt-Tätigkeit meine Passion ist. Und dadurch, dass meine Frau selbst gerne Sport macht, kann sie das auch gut nachvollziehen.

\section{Finden Sie neben Beruf und Familie auch noch Zeit für Ihr Sporttraining?}

Ja, das muss Platz haben. Meist in Verbindung mit dem Arbeitsweg. Entweder fahre ich mit dem Velo oder ich gehe vor der Arbeit Schwimmen oder in der Mittagspause Laufen. Das Morgenschwimmtraining um 6.00 Uhr, wo die Triathleten und Schwimmer trainieren, ist immer ein perfekter Start in den Tag. Da nehme ich dann aber immer die langsame Bahn (lacht).

\section{Was würden Sie einem jungen Kollegen raten, der auch gerne Verbandsarzt werden möchte?} Ich berate meine jungen Kollegen gerne, und es haben sich in der Vergangenheit auch schon einige Assistenzärzte diesbezüglich an mich gewandt. Ich sage dann immer: Nehmt Kontakt auf mit einem älteren Kollegen, der schon Verbandsarzt in der entsprechenden Sportart ist, die euch interessiert. Und gebt euch zumindest am Anfang auch mit einem lokalen Verein zufrieden. Man kann nicht gleich mit der Elite anfangen, sondern man muss Geduld haben und zunächst einmal die Nachwuchssportler betreuen. Dann kommt man auch zum Ziel!

\section{Bildnachweis}

Fotos zVg von Patrik Noack.

Haben Sie als Mediziner auch einen aussergewöhnlichen Beruf, den Sie unseren Lesern gern vorstellen möchten? Dann freuen wir uns auf Ihre Bewerbung per E-Mail an: tkuehnle[at]emh.ch 